TECHNION 96-15

$\mathrm{MZ}-\mathrm{TH} / 96-17$

\title{
Spectator Effects in the Heavy Quark Effective Theory
}

\author{
B. Blok, J. G. Körner, D. Pirjol and J. C. Rojas* \\ Department of Physics, Technion-Israel Institute of Technology, Haifa 32000, Israel and \\ Johannes-Gutenberg Universität, Institut für Physik (ThEP), \\ Staudinger Weg 7, 55099 Mainz, Germany
}

\begin{abstract}
We present a complete analysis of the Heavy Quark Effective Theory Lagrangian at order $1 / \mathrm{m}^{2}$ in the leading logarithmic approximation, including effects induced by spectator quarks. At this order new correction terms appear in the effective Lagrangian, as four-quark operators containing both heavy and light quark fields. We compute the coefficients of these operators in the leadinglogarithmic approximation, and the matching conditions at one-loop order. Two of these operators break the heavy quark spin symmetry, and we estimate their contribution to the hyperfine splitting of the heavy mesons. We find that they make a positive contribution to the hyperfine splitting of about $10 \%$ of the measured splitting in the charm case and of up to $5 \%$ in the bottom case.
\end{abstract}

\footnotetext{
* On leave of absence from the Universidad de Santiago de Chile, Avenida Bernardo O'Higgins 3363, Santiago, Chile
} 


\section{INTRODUCTION}

The heavy mass expansion established itself as a valuable tool for the study of hadrons containing one heavy quark [1, 2, 3]. This expansion is formulated most naturally in terms of an effective theory, the heavy quark effective theory (HQET), which is an approximation to QCD with one heavy quark. The corrections to this approximation are controlled by the small parameter $\Lambda_{Q C D} / m$, where $\Lambda_{Q C D} \simeq 300 \mathrm{MeV}$ is a typical scale of low-energy QCD and $m$ is the heavy quark mass.

The applications of the heavy quark effective theory to physical problems presented so far include correction terms of order $1 / m^{2}$ [4, 5, 6] and in some recent instances even $1 / m^{3}$ 7, 8]. We investigate in this paper the effects induced by spectator quarks in the HQET. They appear first at order $1 / m^{2}$ and are associated in an effective theory language with $4^{-}$ quark operators containing both heavy- and light-quark fields. These operators mix under renormalization with the other operators of dimension six present in the HQET Lagrangian already at tree level, and their inclusion changes the form of the renormalized Lagrangian.

We define the operator basis in Section II and compute their mixing under renormalization. The coefficients of these operators can be obtained from a one-loop matching calculation, which is presented in Section III, together with a leading-log renormalization-group running. As an application we estimate in Section IV their total contribution to the hyperfine splitting of heavy-light mesons in the framework of the factorization approximation.

\section{DIMENSION-6 OPERATORS}

The Lagrangian of the heavy quark effective theory is written as an expansion in the inverse heavy quark mass [1, 2, 3]

$$
\mathcal{L}_{H Q E T}=\bar{h}(i v \cdot D) h+\frac{1}{2 m} \mathcal{L}_{1}+\frac{1}{(2 m)^{2}} \mathcal{L}_{2}+\cdots
$$

The heavy quark field $h$ annihilates static heavy quarks moving with a fixed velocity $v$ and

satisfies the condition $\frac{1}{2}(1+\psi) h=h$. The finite-mass effects appear as interaction terms in the Lagrangian suppressed by powers of $1 / m$. The first two correction terms have the 
well-known expressions [9, 10, 11, 12, 13]

$$
\begin{aligned}
\mathcal{L}_{1} & =c_{k}(\mu) \bar{h}(i D)^{2} h+c_{m}(\mu) \bar{h} \frac{g}{2} \sigma_{\mu \nu} F^{\mu \nu} h+c_{e}(\mu) \bar{h}(i v \cdot D)^{2} h \\
\mathcal{L}_{2} & =c_{D}(\mu) \mathcal{O}_{D}+c_{S O}(\mu) \mathcal{O}_{S O} .
\end{aligned}
$$

The coefficients of the operators in Eq. (21) are known in the leading logarithmic approximation [10, 11]

$$
c_{k}(\mu)=1, \quad c_{m}(\mu)=-\left(\frac{\alpha_{s}(\mu)}{\alpha_{s}(m)}\right)^{-3 / \beta_{0}}, \quad c_{e}(\mu)=2-3\left(\frac{\alpha_{s}(\mu)}{\alpha_{s}(m)}\right)^{-8 /\left(3 \beta_{0}\right)}
$$

where $\beta_{0}=11-\frac{2}{3} n_{f}$ is the one-loop coefficient of the beta function for $n_{f}$ active quarks. The coefficient of the last operator in (2) is gauge-dependent; the value quoted above corresponds to the Feynman gauge.

To order $1 / \mathrm{m}^{2}$ there are two local operators contributing at tree-level, the Darwin term and the spin-orbit interaction energy respectively

$$
\begin{aligned}
\mathcal{O}_{D} & =\frac{g}{2}\left(D^{\mu} F_{\mu \nu}^{a}\right)\left(\bar{h} v_{\nu} t^{a} h\right) \\
\mathcal{O}_{S O} & =i g\left(\bar{h} \sigma_{\alpha \nu} v_{\mu} F^{\mu \nu} D^{\alpha} h\right)+\frac{i g}{2}\left(D_{\alpha} F_{\mu \nu}^{a}\right)\left(\bar{h} \sigma^{\alpha \nu} v^{\mu} t^{a} h\right) .
\end{aligned}
$$

The coefficients $c_{D}(\mu)$ and $c_{S O}(\mu)$ were calculated in [13] (see also [14, 15]) using a different operator basis and in the absence of light quarks. We reconsider here their calculation in a basis more suited for our present purposes, with a different result for $c_{D}(\mu)$.

In addition to the operators shown in (3), there are three other types of dimension-6 operators which must be added to $\mathcal{L}_{2}$ :

a) the gluonic operators

$$
\mathcal{O}_{F^{3}}=\frac{1}{4} g f_{a b c} F_{\mu \nu}^{a} F_{\nu \lambda}^{b} F_{\lambda \mu}^{c}, \quad \mathcal{O}_{(D F)^{2}}=\frac{1}{2}\left(D^{\mu} F_{\mu \nu}^{a}\right)\left(D_{\lambda} F^{a \lambda \nu}\right) .
$$

A third gluonic operator $F_{\mu \nu}^{a} D^{2} F^{a \mu \nu}$ has been eliminated in zero momentum insertions with the help of the Bianchi identity, which gives

$$
\int \mathrm{d}^{4} x\left(4 \mathcal{O}_{F^{3}}+\mathcal{O}_{(D F)^{2}}+\frac{1}{4} F_{\mu \nu}^{a} D^{2} F^{a \mu \nu}\right)=0
$$

Their coefficients have been computed many times [13, 17, 18]. At one-loop order they are given by

$$
c_{F^{3}}(m)=\frac{\alpha_{s}(m)}{45 \pi}, \quad c_{(D F)^{2}}(m)=-\frac{2 \alpha_{s}(m)}{15 \pi}
$$


At one-loop order $\mathcal{O}_{F^{3}}$ does not mix with other dimension-6 operators and will be neglected in the following. The second operator $\mathcal{O}_{(D F)^{2}}$ will be eliminated in favor of 4-quark operators using the equation of motion of the gluon field (see Eq. (43) below).

b) Operators which vanish by the equation of motion of the heavy quark field $i v \cdot D h=0$. Even though their expectation values vanish, they can contribute when considering mass corrections to the matrix elements of currents. A discussion of their renormalization is given in Appendix A.

c) 4-quark operators built out of heavy-light and light-light quark fields. It is these operators which will be the main point of interest of this paper.

There are four independent heavy-light 4-quark operators, which can be conveniently chosen as follows

$$
\begin{aligned}
\mathcal{O}_{1}^{h l} & =\frac{g^{2}}{2} \sum_{q}\left(\bar{q} \gamma_{\mu} t^{a} q\right)\left(\bar{h} \gamma^{\mu} t^{a} h\right) \\
\mathcal{O}_{2}^{h l} & =\frac{g^{2}}{2} \sum_{q}\left(\bar{q} \gamma_{\mu} \gamma_{5} t^{a} q\right)\left(\bar{h} \gamma^{\mu} \gamma_{5} t^{a} h\right) \\
\mathcal{O}_{3}^{h l} & =\frac{g^{2}}{2} \sum_{q}\left(\bar{q} \gamma_{\mu} q\right)\left(\bar{h} \gamma^{\mu} h\right) \\
\mathcal{O}_{4}^{h l} & =\frac{g^{2}}{2} \sum_{q}\left(\bar{q} \gamma_{\mu} \gamma_{5} q\right)\left(\bar{h} \gamma^{\mu} \gamma_{5} h\right) .
\end{aligned}
$$

The summation extends over $n_{f}$ dynamic quarks, for example $n_{f}=4(q=u, d, s, c)$ for the case of a heavy bottom quark.

The equation of motion for the gluon field gives a relation among the Darwin term $\mathcal{O}_{D}$ Eq. (15) and one of the 4-quark operators $\mathcal{O}_{i}^{h l}$

$$
\mathcal{O}_{D}=\mathcal{O}_{1}^{h l}+\frac{g^{2}}{2}\left(\bar{h} t^{a} h\right)\left(\bar{h} t^{a} h\right)
$$

The structure of the possible 4-quark operators containing only light quark fields is similar to that of the heavy-light operators. There are altogether 10 such operators, which can be divided into three groups with different transformation properties under $S U\left(n_{f}\right)_{L} \times S U\left(n_{f}\right)_{R}$. The operators in each group mix only among themselves under renormalization. Their renormalization has been discussed in detail in Refs. [20, 21, 22]. Only one group of operators 
is relevant for our case, which can be chosen as

$$
\begin{aligned}
\mathcal{O}_{1}^{l l} & =\frac{g^{2}}{2} \sum_{q, q^{\prime}}\left(\bar{q} \gamma_{\mu} t^{a} q\right)\left(\bar{q} \gamma^{\mu} t^{a} q\right) \\
\mathcal{O}_{2}^{l l} & =\frac{g^{2}}{2} \sum_{q, q^{\prime}}\left(\bar{q} \gamma_{\mu} \gamma_{5} t^{a} q\right)\left(\bar{q} \gamma^{\mu} \gamma_{5} t^{a} q\right) \\
\mathcal{O}_{3}^{l l} & =\frac{g^{2}}{2} \sum_{q, q^{\prime}}\left(\bar{q} \gamma_{\mu} q\right)\left(\bar{q} \gamma^{\mu} q\right) \\
\mathcal{O}_{4}^{l l} & =\frac{g^{2}}{2} \sum_{q, q^{\prime}}\left(\bar{q} \gamma_{\mu} \gamma_{5} q\right)\left(\bar{q} \gamma^{\mu} \gamma_{5} q\right) .
\end{aligned}
$$

The complete basis of the dimension-6 operators includes also three nonlocal operators consisting of time-ordered products of dimension- 5 operators

$$
\begin{aligned}
\mathcal{O}_{k k} & =\frac{i}{2} \int \mathrm{d}^{4} x \mathrm{~T}\left[\bar{h}(i D)^{2} h\right](x)\left[\bar{h}(i D)^{2} h\right](0) \\
\mathcal{O}_{k m} & =i \int \mathrm{d}^{4} x \mathrm{~T}\left[\bar{h}(i D)^{2} h\right](x)\left[\bar{h} \frac{g}{2} \sigma \cdot F h\right](0) \\
\mathcal{O}_{m m} & =\frac{i}{2} \int \mathrm{d}^{4} x \mathrm{~T}\left[\bar{h} \frac{g}{2} \sigma \cdot F h\right](x)\left[\bar{h} \frac{g}{2} \sigma \cdot F h\right](0) .
\end{aligned}
$$

We will use a compact vector notation for the thirteen operators (56]10,21), defined as follows

$$
\hat{\mathcal{O}}=\left(\begin{array}{l}
\mathcal{H} \\
\mathcal{O}^{h l} \\
\mathcal{O}^{l l}
\end{array}\right), \quad \text { with } \mathcal{H}=\left(\begin{array}{c}
\mathcal{O}_{D} \\
\mathcal{O}_{S O} \\
\mathcal{O}_{k k} \\
\mathcal{O}_{k m} \\
\mathcal{O}_{m m}
\end{array}\right), \quad \hat{\mathcal{O}}^{h l}=\left(\begin{array}{c}
\mathcal{O}_{1}^{h l} \\
\mathcal{O}_{2}^{h l} \\
\mathcal{O}_{3}^{h l} \\
\mathcal{O}_{4}^{h l}
\end{array}\right), \quad \hat{\mathcal{O}}^{l l}=\left(\begin{array}{c}
\mathcal{O}_{1}^{l l} \\
\mathcal{O}_{2}^{l l} \\
\mathcal{O}_{3}^{l l} \\
\mathcal{O}_{4}^{l l}
\end{array}\right)
$$

and a corresponding vector notation for their Wilson coefficients $c_{i}(\mu)$, carrying the same index as the operators $\mathcal{O}_{i}$. Requiring that the total renormalized Lagrangian $\mathcal{L}_{2}$ be scaleindependent gives a renormalization-group equation for the coefficients $\hat{c}(\mu)$

$$
\mu \frac{\mathrm{d}}{\mathrm{d} \mu} \hat{c}-\hat{\gamma}^{T} \hat{c}=0 .
$$

The anomalous dimension matrix can be written as

$$
\hat{\gamma}=\left(\begin{array}{ccc}
A & B & 0 \\
C & D & 0 \\
0 & E & F
\end{array}\right) \text {. }
$$


To one-loop order the blocks in this matrix take the values

$$
\begin{aligned}
& A=\frac{g^{2}}{(4 \pi)^{2}}\left(\begin{array}{ccccc}
4 & 0 & 0 & 0 & 0 \\
0 & 0 & 0 & 0 & 0 \\
-\frac{146}{9} & 0 & 0 & 0 & 0 \\
0 & -12 & 0 & 6 & 0 \\
-10 & 0 & 0 & 0 & 12
\end{array}\right) \\
& B=\frac{g^{2}}{(4 \pi)^{2}}\left(\begin{array}{cccc}
9 & 0 & 0 & 0 \\
0 & -5 & 0 & -\frac{8}{3} \\
-18 & 0 & 0 & 0 \\
0 & -\frac{40}{3} & 0 & -\frac{64}{9} \\
9 & -\frac{5}{3} & 0 & -\frac{8}{9}
\end{array}\right) \\
& C=\frac{g^{2}}{(4 \pi)^{2}}\left(\begin{array}{ccccc}
\frac{4}{3} n_{f} & 0 & 0 & 0 & 0 \\
0 & 0 & 0 & 0 & 0 \\
0 & 0 & 0 & 0 & 0 \\
0 & 0 & 0 & 0 & 0
\end{array}\right) \\
& D=\frac{g^{2}}{(4 \pi)^{2}} \operatorname{diag}\left(13-\frac{4}{3} n_{f}, 13-\frac{4}{3} n_{f}, 22-\frac{4}{3} n_{f}, 22-\frac{4}{3} n_{f}\right), \\
& E=\frac{g^{2}}{(4 \pi)^{2}}\left(\begin{array}{cccc}
-\frac{4}{9}+\frac{8}{3} n_{f} & 0 & 0 & 0 \\
-\frac{4}{9} & 0 & 0 & 0 \\
\frac{8}{3} & 0 & 0 & 0 \\
\frac{8}{3} & 0 & 0 & 0
\end{array}\right) \\
& F=\frac{g^{2}}{(4 \pi)^{2}}\left(\begin{array}{cccc}
\frac{113}{9}+\frac{4}{3} n_{f} & 5 & 0 & \frac{8}{3} \\
\frac{41}{9} & 13-\frac{4}{3} n_{f} & \frac{8}{3} & 0 \\
\frac{8}{3} & 12 & 22-\frac{4}{3} n_{f} & 0 \\
\frac{44}{3} & 0 & 0 & 22-\frac{4}{3} n_{f}
\end{array}\right) .
\end{aligned}
$$

The blocks $D$ and $F$ contain contributions which take into account the running of the $g$ factors contained in the definition of the four-quark operators. We have implicitly used the fact established in [13] that the form of $\mathcal{O}_{S O}$ as given in (6) is preserved under renormalization. We agree with [15] on the renormalization of the Darwin term in $A$ in the absence of 
the mixing with the four-quark operators. The lower diagonal block $F$ has been calculated previously in 18]. (Note added. After correcting the entries $C_{11}, D_{11}, E_{11}$ we agree with the results of Ref. [30]. These changes affect only the result for the Darwin term $c_{D}(\mu)$, but leave all the other results of the paper unchanged.)

\section{MATCHING AND RUNNING}

The coefficients of the operators $\mathcal{H}$ in Eq. (22) are given, at the matching scale $\mu=$ $m$, by their tree-level values $c_{\mathcal{H}}(m)=\operatorname{column}(1,1,1,-1,1)$. The coefficients of the nonlocal operators (19,21) are given simply by the products of the respective local dimension-5 operators

$$
\begin{aligned}
c_{k k}(\mu) & =1 \\
c_{k m}(\mu) & =-\left(\frac{\alpha_{s}(\mu)}{\alpha_{s}(m)}\right)^{-3 / \beta_{0}} \\
c_{m m}(\mu) & =\left(\frac{\alpha_{s}(\mu)}{\alpha_{s}(m)}\right)^{-6 / \beta_{0}}
\end{aligned}
$$

Integration of the RGE for the Wilson coefficient of the Darwin operator $c_{D}(\mu)$ is complicated by the mixing between $\mathcal{O}_{D}$ and $\mathcal{O}_{1}^{h l}$. The initial conditions for the leading log running of the Wilson coefficients of the 4-quark operators are $c_{i}^{h l}(m)=0$. We compute below also the leading nonvanishing contribution to the matching of these Wilson coefficients, of order $\alpha_{s}(m)$ (see Eqs. (152)-(155)).

We start by solving the RGE for the coefficients $\hat{C}(\mu)=\left(c_{D}(\mu), c_{1}^{h l}(\mu)\right)$, which has the form

$$
\mu \frac{d}{d \mu} \hat{C}(\mu)=A_{2}^{T} \hat{C}(\mu)+\hat{B}(\mu)
$$

with $A_{2}^{T}$ the corresponding $2 \times 2$ block of the anomalous dimension matrix $\hat{\gamma}$ in Eq. (24), and $\hat{B}(\mu)$ an inhomogeneous term

$$
A_{2}^{T}=\frac{\alpha_{s}}{4 \pi}\left(\begin{array}{cc}
4 & \frac{4}{3} n_{f} \\
9 & 13-\frac{4}{3} n_{f}
\end{array}\right), \quad \hat{B}(\mu)=\frac{\alpha_{s}}{4 \pi}\left(\begin{array}{c}
-\frac{146}{9}-10 z^{-6 / \beta_{0}} \\
-18+9 z^{-6 / \beta_{0}}
\end{array}\right)
$$

We denoted here $z=\alpha_{s}(\mu) / \alpha_{s}(m)$, and we neglected contributions to $\hat{B}(\mu)$ from the lightlight 4-quark operators $\mathcal{O}_{i}^{l l}$. These contributions are of the form $\alpha_{s}\left(\alpha_{s} \log (\mu / m)\right)^{n}$, and 
are thus formally of higher order than the leading logs computed here. They are however calculable, see below.

The matrix $A_{2}^{T}$ is diagonalized as

$$
U^{-1} A_{2}^{T} U=\frac{\alpha_{s}}{4 \pi}\left(\begin{array}{cc}
\gamma_{1} & 0 \\
0 & \gamma_{2}
\end{array}\right), \quad \text { with } \quad U=\left(\begin{array}{cc}
\frac{4}{27} n_{f} & -1 \\
1 & 1
\end{array}\right), \quad U^{-1}=\frac{1}{R}\left(\begin{array}{cc}
1 & 1 \\
-1 & \frac{4}{27} n_{f}
\end{array}\right)
$$

with $\gamma_{1}=13, \gamma_{2}=4-\frac{4}{3} n_{f}$ and $R=1+\frac{4}{27} n_{f}$. Redefining the Wilson coefficients as $\hat{C}(\mu)=U \hat{C}^{\prime}(\mu)$, the RGEs for the components of $\hat{C}^{\prime}(\mu)$ are decoupled

$$
\mu \frac{d}{d \mu} \hat{C}^{\prime}(\mu)=U^{-1} A_{2}^{T} U \hat{C}^{\prime}(\mu)+U^{-1} \hat{B}(\mu)
$$

and can be solved by simple integration.

Solving the RGE for the Wilson coefficients $c_{D}(\mu), c_{1}^{h l}(\mu)$, one has to distinguish the two cases i) $n_{f} \neq 3$, and ii) $n_{f}=3$ separately, corresponding to $\gamma_{2}$ being nonvanishing, and vanishing, respectively.

The solutions for the case i) $n_{f} \neq 3$ are given by

$$
\begin{aligned}
c_{D}(\mu)=\frac{1}{R}\{ & \frac{16 n_{f}^{2}+96 n_{f}-81}{108\left(n_{f}+6\right)} z^{-6 / \beta_{0}}-\frac{1232}{3159} n_{f} z^{-\gamma_{1} /\left(2 \beta_{0}\right)} \\
& \left.+\frac{12 n_{f}^{2}+353 n_{f}+1605}{12\left(n_{f}-3\right)\left(n_{f}+6\right)} z^{-\gamma_{2} /\left(2 \beta_{0}\right)}+\frac{77\left(16 n_{f}^{2}-48 n_{f}-1053\right.}{3159\left(n_{f}-3\right)}\right\} \\
c_{1}^{h l}(\mu)=\frac{1}{R}\left\{\frac{4 n_{f}+27}{4\left(n_{f}+6\right)} z^{-6 / \beta_{0}}+\frac{308}{117} z^{-\gamma_{1} /\left(2 \beta_{0}\right)}\right. & \\
& \left.-\frac{12 n_{f}^{2}+353 n_{f}+1605}{12\left(n_{f}-3\right)\left(n_{f}+6\right)} z^{-\gamma_{2} /\left(2 \beta_{0}\right)}-\frac{77\left(4 n_{f}-51\right.}{117\left(n_{f}-3\right)}\right\}
\end{aligned}
$$

The corresponding results for case ii) $n_{f}=3$ are

$$
\begin{aligned}
& c_{D}(\mu)=\frac{1}{R}\left\{\frac{13}{36} z^{-6 / \beta_{0}}-\frac{1232}{1053} z^{-13 /\left(2 \beta_{0}\right)}+\frac{154}{9 \beta_{0}} \log z+\frac{9491}{4212}\right\} \\
& c_{1}^{h l}(\mu)=\frac{1}{R}\left\{\frac{13}{12} z^{-6 / \beta_{0}}-\frac{308}{117} z^{-13 /\left(2 \beta_{0}\right)}-\frac{154}{9 \beta_{0}} \log z+\frac{725}{468}\right\}
\end{aligned}
$$

For both cases i) and ii), the sum of the two Wilson coefficients is given by a common expression

$$
c_{D}(\mu)+c_{1}^{h l}(\mu)=z^{-6 / \beta_{0}}-\frac{308}{117} z^{-13 /\left(2 \beta_{0}\right)}+\frac{308}{117}
$$

This combination corresponds to the coefficient of the Darwin operator in Ref. [30], in a basis in which the 4-quark operator $\mathcal{O}_{1}^{h l}$ is eliminated using the equation of motion Eq. (14). The result Eq. (42) agrees with Eq. (19) of Ref. [30] [31]. 
The results for $c_{D}(\mu), c_{1}^{h l}(\mu)$ presented above neglect mixing into $\mathcal{O}_{1}^{h l}$ from the light-light 4-quark operators $\mathcal{O}_{i}^{l l}$. As mentioned above, they are formally next-to-leading order, and are comparable with other contributions which can not be yet fully computed. They require the one-loop matching of the Darwin operator (obtained in Ref. 23]), and the 2-loop anomalous dimension $A_{2}^{T}$ (not yet available). However, the mixing of $c_{i}^{l l}$ into $c_{D}(\mu), c_{1}^{h l}(\mu)$ is a well defined effects and can be computed from our results.

We present next the Wilson coefficients $c_{i}^{l l}$ in leading log approximation. The equation of motion for the gluon field relates the operator $\mathcal{O}_{(D F)^{2}}$ to the 4-quark operators

$$
\mathcal{O}_{(D F)^{2}}=\frac{1}{2}\left(D^{\mu} F_{\mu \nu}^{a}\right)\left(D_{\lambda} F^{a \lambda \nu}\right)=2 \mathcal{O}_{1}^{h l}+\mathcal{O}_{1}^{l l}+\frac{g^{2}}{2}\left(\bar{h} t^{a} h\right)\left(\bar{h} t^{a} h\right) .
$$

We will use this relation to eliminate the gluonic operator in favor of the 4-quark operators $\mathcal{O}_{1}^{h l}$ and $\mathcal{O}_{1}^{l l}$. Using Eq. (9), this gives the initial condition for $c_{1}^{l l}(m)$

$$
c_{1}^{l l}(m)=-\frac{2 \alpha_{s}(m)}{15 \pi} .
$$

It is now straightforward to solve the RGE for the coefficients $c_{i}^{l l}(\mu)$. We focus on the physically interesting case of the $1 / m_{c}^{2}$ corrections, and take as thus $n_{f}=3$. The results are given by (with $\beta_{0}=9$ )

$$
\begin{aligned}
& c_{1}^{l l}(\mu)=\left(0.0246 z^{-\delta_{1} /\left(2 \beta_{0}\right)}+0.4359 z^{-\delta_{2} /\left(2 \beta_{0}\right)}+0.1646 z^{-\delta_{3} /\left(2 \beta_{0}\right)}+0.3748 z^{-\delta_{4} /\left(2 \beta_{0}\right)}\right) c_{1}^{l l}(m) \\
& c_{2}^{l l}(\mu)=\left(-0.0434 z^{-\delta_{1} /\left(2 \beta_{0}\right)}+0.1961 z^{-\delta_{2} /\left(2 \beta_{0}\right)}-0.3764 z^{-\delta_{3} /\left(2 \beta_{0}\right)}+0.2237 z^{-\delta_{4} /\left(2 \beta_{0}\right)}\right) c_{1}^{l l}(m) \\
& c_{3}^{l l}(\mu)=\left(-0.051 z^{-\delta_{1} /\left(2 \beta_{0}\right)}+0.0767 z^{-\delta_{2} /\left(2 \beta_{0}\right)}+0.0741 z^{-\delta_{3} /\left(2 \beta_{0}\right)}-0.0998 z^{-\delta_{4} /\left(2 \beta_{0}\right)}\right) c_{1}^{l l}(m) \\
& c_{4}^{l l}(\mu)=\left(0.0290 z^{-\delta_{1} /\left(2 \beta_{0}\right)}+0.1706 z^{-\delta_{2} /\left(2 \beta_{0}\right)}-0.0324 z^{-\delta_{3} /\left(2 \beta_{0}\right)}-0.1672 z^{-\delta_{4} /\left(2 \beta_{0}\right)}\right) c_{1}^{l l}(m)
\end{aligned}
$$

The eigenvalues of the mixing matrix $F$ are given by $\alpha_{s} /(4 \pi) \delta_{i}$, with $\delta_{1}=20.2680, \delta_{2}=$ $24.8138, \delta_{3}=4.4516, \delta_{4}=12.0222$.

We turn next to the spin symmetry breaking operators. We reproduce the result for the Wilson coefficient of the spin-orbit operator $\mathcal{O}_{S O}$ obtained in Ref. [13]

$$
c_{S O}(\mu)=2\left(\frac{\alpha_{s}(\mu)}{\alpha_{s}(m)}\right)^{-3 / \beta_{0}}-1 .
$$

This coefficient has been also obtained without an explicit calculation in [14] from reparametrization invariance (RPI) arguments 16]. No such RPI constraints exist for the 
coefficient of the Darwin term $c_{D}$, contrary to the argument of Ref. 14]. A discussion of the RPI constraints for the renormalized $1 / m^{2}$ HQET Lagrangian is given in Appendix B.

Finally, the coefficients of the remaining heavy-light four-quark operators (11) 13) are found to be

$$
\begin{aligned}
c_{2}^{h l}(\mu)= & -\frac{10}{21-4 n_{f}} z^{-3 / \beta_{0}}+\frac{5}{3-4 n_{f}} z^{-6 / \beta_{0}}-\frac{15}{39-4 n_{f}} \\
& +\frac{20\left(99+96 n_{f}-16 n_{f}^{2}\right)}{\left(4 n_{f}-39\right)\left(4 n_{f}-21\right)\left(4 n_{f}-3\right)} z^{-\left(13-\frac{4}{3} n_{f}\right) /\left(2 \beta_{0}\right)} \\
c_{3}^{h l}(\mu)= & 0 \\
c_{4}^{h l}(\mu)= & -\frac{4}{3\left(12-n_{f}\right)} z^{-3 / \beta_{0}}+\frac{4}{3\left(15-2 n_{f}\right)} z^{-6 / \beta_{0}}-\frac{4}{33-2 n_{f}} \\
& +\frac{4\left(-639+156 n_{f}-8 n_{f}^{2}\right)}{3\left(n_{f}-12\right)\left(2 n_{f}-33\right)\left(2 n_{f}-15\right)} z^{-\left(22-\frac{4}{3} n_{f}\right) /\left(2 \beta_{0}\right)} .
\end{aligned}
$$

A full one-loop determination of the coefficients $c_{i}^{h l}(\mu)$ requires an explicit matching calculation. This involves computing the box diagrams for heavy-light quark scattering in QCD shown in Fig.1. When expanded in powers of $1 / m$ the total result for these diagrams is

$$
\begin{aligned}
I_{Q C D} & =\frac{i g^{4}}{(4 \pi)^{2}}\left\{-\frac{3}{2 \lambda^{2}}\left[\gamma_{\mu} t^{a}\right]_{\beta \alpha}\left[\gamma^{\mu} t^{a}\right]_{\delta \gamma}+\frac{1}{m}\left[-\frac{3 \pi}{4 \lambda}\left[\gamma_{\mu} t^{a}\right]_{\beta \alpha}\left[\gamma^{\mu} t^{a}\right]_{\delta \gamma}+\frac{5 \pi}{9 \lambda}\left[\gamma_{\mu} \gamma_{5} t^{a}\right]_{\beta \alpha}\left[\gamma^{\mu} \gamma_{5} t^{a}\right]_{\delta \gamma}\right.\right. \\
+ & \left.\frac{8 \pi}{27 \lambda}\left[\gamma_{\mu} \gamma_{5}\right]_{\beta \alpha}\left[\gamma^{\mu} \gamma_{5}\right]_{\delta \gamma}\right]+\frac{1}{m^{2}}\left[\frac{9}{4}\left[\gamma_{\mu} t^{a}\right]_{\beta \alpha}\left[\gamma^{\mu} t^{a}\right]_{\delta \gamma}+\frac{5}{12}\left(\ln \frac{\lambda^{2}}{m^{2}}-2\right)\left[\gamma_{\mu} \gamma_{5} t^{a}\right]_{\beta \alpha}\left[\gamma^{\mu} \gamma_{5} t^{a}\right]_{\delta \gamma}\right. \\
+ & \left.\left.\frac{2}{9}\left(\ln \frac{\lambda^{2}}{m^{2}}-2\right)\left[\gamma_{\mu} \gamma_{5}\right]_{\beta \alpha}\left[\gamma^{\mu} \gamma_{5}\right]_{\delta \gamma}\right]+\mathcal{O}\left(1 / m^{3}\right)\right\}
\end{aligned}
$$

To simplify the calculation we have taken a massless light quark scattering in the forward direction. The infrared singularities have been regulated with a finite gluon mass $\lambda$.

A computation of the same diagrams using the effective theory Feynman rules for the heavy line with insertions of all $1 / m$ corrections up to second order gives, in the $\overline{M S}$ scheme,

$$
\begin{aligned}
I_{H Q E T} & =\frac{i g^{4}}{(4 \pi)^{2}}\left\{-\frac{3}{2 \lambda^{2}}\left[\gamma_{\mu} t^{a}\right]_{\beta \alpha}\left[\gamma^{\mu} t^{a}\right]_{\delta \gamma}+\frac{1}{m}\left[-\frac{3 \pi}{4 \lambda}\left[\gamma_{\mu} t^{a}\right]_{\beta \alpha}\left[\gamma^{\mu} t^{a}\right]_{\delta \gamma}+\frac{5 \pi}{9 \lambda}\left[\gamma_{\mu} \gamma_{5} t^{a}\right]_{\beta \alpha}\left[\gamma^{\mu} \gamma_{5} t^{a}\right]_{\delta \gamma}\right.\right. \\
+ & \left.\frac{8 \pi}{27 \lambda}\left[\gamma_{\mu} \gamma_{5}\right]_{\beta \alpha}\left[\gamma^{\mu} \gamma_{5}\right]_{\delta \gamma}\right]+\frac{1}{m^{2}}\left[\frac{5}{12}\left(\ln \frac{\lambda^{2}}{\mu^{2}}+2\right)\left[\gamma_{\mu} \gamma_{5} t^{a}\right]_{\beta \alpha}\left[\gamma^{\mu} \gamma_{5} t^{a}\right]_{\delta \gamma}\right. \\
+ & \left.\left.\frac{2}{9}\left(\ln \frac{\lambda^{2}}{\mu^{2}}+2\right)\left[\gamma_{\mu} \gamma_{5}\right]_{\beta \alpha}\left[\gamma^{\mu} \gamma_{5}\right]_{\delta \gamma}\right]+\mathcal{O}\left(1 / m^{3}\right)\right\}
\end{aligned}
$$

The first two terms in the $1 / m$ expansion agree identically, as does the logarithmic dependence on the infrared regulator $\lambda$ in the $1 / m^{2}$ part. Imposing equality of these two 
expressions requires adding the four-quark operators (10, 13) to the effective theory with coefficients

$$
\begin{aligned}
c_{1}^{h l}(\mu) & =\frac{18 g^{2}}{(4 \pi)^{2}} \\
c_{2}^{h l}(\mu) & =\frac{g^{2}}{(4 \pi)^{2}}\left(\frac{10}{3} \log \frac{\mu^{2}}{m^{2}}-\frac{40}{3}\right) \\
c_{3}^{h l}(\mu) & =0 \\
c_{4}^{h l}(\mu) & =\frac{g^{2}}{(4 \pi)^{2}}\left(\frac{16}{9} \log \frac{\mu^{2}}{m^{2}}-\frac{64}{9}\right) .
\end{aligned}
$$

The logarithmic terms agree, as they should, with the leading terms obtained by expanding the LLA expressions Eqs. (39), (47)-(49).

One should point out that the constant terms in the full one-loop expressions for these coefficients are scheme-dependent. The $\mathcal{O}\left(\alpha_{s}\right)$ terms become well-defined only after renormalization-group improvement. In this process the renormalization scheme dependence of the constant terms in (52,55) is cancelled by the scheme dependence of a two-loop anomalous dimension [24]. However, there are reasons to expect the partial results (52 [55) to provide a good estimate of the full $\mathcal{O}\left(\alpha_{s}\right)$ correction, based on empirical evidence [25] that the contribution of the two-loop anomalous dimension is often much smaller than the constant term in the one-loop expression of the coefficient.

On the other hand, the logarithmic terms in (52 [55) and the LLA sums (39474.49) are scheme independent. However, the use of the leading-log resummed expression is appropriate only when the logarithmic terms in (52,55) dominate clearly over the constant terms. If this

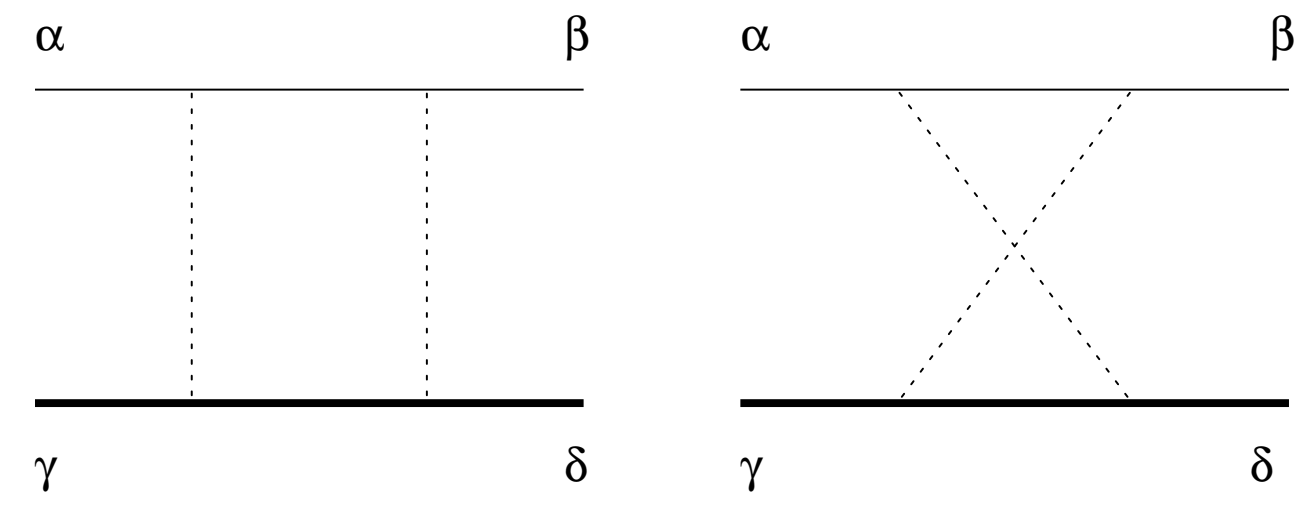

FIG. 1: Diagrams contributing to heavy-light quark scattering which are needed for the matching conditions for the four-quark operators $c_{i}^{h l}(m)$. The thick line denotes a heavy quark and the dotted lines denote gluons. 
is not the case, then the use of the full one-loop results (52,55), with all the above limitations, is likely to be a better approximation to the true value of the Wilson coefficient. Such a situation is encountered in the case of the charm quark heavy mass expansion, as discussed in the next section.

\section{APPLICATION: HEAVY MESONS HYPERFINE SPLITTING}

The importance of considering the four-quark operators (10,13) becomes immediately apparent if one notes that the equation of motion for the gluon field gives $\mathcal{O}_{D}=\mathcal{O}_{1}^{h l}$. The coefficients of these two operators (38) and (39) get contributions of the same order, such that neglecting $\mathcal{O}_{1}^{h l}$ would lead to an incomplete result. Adding the contribution of $\mathcal{O}_{1}^{h l}$ together with that of the Darwin term gives, in the leading logarithmic approximation, the "effective" coefficient Eq. (42) for the local operator of $O\left(1 / m^{2}\right)$

$$
c_{D}(\mu)=\left(\frac{\alpha_{s}(\mu)}{\alpha_{s}(m)}\right)^{-6 / \beta_{0}}-\frac{308}{117}\left[\left(\frac{\alpha_{s}(\mu)}{\alpha_{s}(m)}\right)^{-13 /\left(2 \beta_{0}\right)}-1\right] .
$$

It is possible to eliminate $\mathcal{O}_{1}^{h l}$ from the effective Lagrangian to all orders in $\alpha_{s}$, in favor of the Darwin operator, which contributes with coefficient $c_{D}(\mu)+c_{1}^{h l}(\mu) \rightarrow c_{D}(\mu)[30]$.

More interesting from a practical point of view is the fact that the operators $\mathcal{O}_{2}^{h l}$ and $\mathcal{O}_{4}^{h l}$ break the heavy quark spin symmetry and thus contribute to the hyperfine splittings of the heavy hadrons. The remainder of this Section is dedicated to a discussion of these effects.

We first briefly review the derivation of the mass formula for the mass of a heavy hadron, including the contributions of the 4-quark operators (10)-(13). The mass of a heavy hadron can be written as an expansion in inverse powers of the heavy quark mass as [5, 6 ]

$$
m_{B^{(*)}}=m_{b}+\bar{\Lambda}+\left\langle\mathrm{TH}_{\text {int }} \exp \left(-i \int \mathrm{d}^{4} x \mathcal{H}_{\text {int }}(x)\right)\right\rangle
$$

with $\mathcal{H}_{\text {int }}=-\mathcal{L}_{\text {int }}=-\frac{1}{2 m_{b}} \mathcal{L}_{1}-\cdots$ the interaction Hamiltonian. The expectation value is taken between eigenstates of the leading order HQET Lagrangian which have the mass $m_{b}+\bar{\Lambda}$. This gives, including terms up to order $1 / m_{b}^{2}$,

$$
\begin{aligned}
m_{B^{(*)}} & =m_{b}+\bar{\Lambda}-\frac{1}{2 m_{b}}\left\langle\mathcal{L}_{1}\right\rangle-\frac{1}{\left(2 m_{b}\right)^{2}}\left(\left\langle\mathcal{L}_{2}\right\rangle+2\left(c_{k}(\mu)\right)^{2}\left\langle\mathcal{O}_{k k}\right\rangle\right. \\
& \left.+c_{k}(\mu) c_{m}(\mu)\left\langle\mathcal{O}_{k m}\right\rangle+2\left(c_{m}(\mu)\right)^{2}\left\langle\mathcal{O}_{m m}\right\rangle+\sum_{i=1}^{4} c_{i}^{h l}(\mu)\left\langle\mathcal{O}_{i}^{h l}\right\rangle\right)+\cdots
\end{aligned}
$$


The expectation values appearing in this formula can be parametrized as (using the notations of Ref. [6])

$$
\begin{aligned}
\left\langle\mathcal{L}_{1}\right\rangle & =-c_{k}(\mu) \mu_{\pi}^{2}-c_{m}(\mu) \frac{d_{H}}{3} \mu_{G}^{2} \\
\left\langle\mathcal{L}_{2}\right\rangle & =c_{D}(\mu) \mu_{D}^{3}+\frac{d_{H}}{3} c_{S O}(\mu) \mu_{S O}^{3} \\
\left\langle\mathcal{O}_{k k}\right\rangle & =\mu_{\pi \pi}^{3} \\
\left\langle\mathcal{O}_{k m}\right\rangle & =\frac{d_{H}}{3} \mu_{\pi G}^{3} \\
\left\langle\mathcal{O}_{m m}\right\rangle & =\mu_{G G}^{3}+\frac{1}{2} \rho_{S}^{3}+\frac{1}{6} d_{H} \rho_{A}^{3},
\end{aligned}
$$

with $d_{H}=3$ for a pseudoscalar meson and -1 for a vector meson.

The matrix elements of the four-quark operators (10)-(13) can be estimated with the help of the factorization approximation [26]. (An alternative model-independent determination of $\mathcal{O}_{1}^{h l}$ (based on a method proposed in [27]) has been given in Ref. [28].) This is done by first applying a Fierz transformation to bring the operator into the form $\left(\bar{h} \Gamma^{\prime} q\right)\left(\bar{q} \Gamma^{\prime} h\right)$, after which the vacuum state is inserted between the two currents. Only color singlet currents will give a nonvanishing contribution. In this way we obtain

$$
\begin{aligned}
& \frac{1}{2 m_{B}}\left\langle\bar{B}(v)\left|\frac{g^{2}}{2} \sum_{q}\left(\bar{q} \gamma_{\mu} \gamma_{5} t^{a} q\right)\left(\bar{h} \gamma^{\mu} \gamma_{5} t^{a} h\right)\right| \bar{B}(v)\right\rangle=-\frac{2 \pi \alpha_{s}}{3} f_{B}^{2} m_{B} \\
& \frac{1}{2 m_{B}}\left\langle\bar{B}(v)\left|\frac{g^{2}}{2} \sum_{q}\left(\bar{q} \gamma_{\mu} \gamma_{5} q\right)\left(\bar{h} \gamma^{\mu} \gamma_{5} h\right)\right| \bar{B}(v)\right\rangle=-\frac{\pi \alpha_{s}}{2} f_{B}^{2} m_{B} \\
& \frac{1}{2 m_{B}}\left\langle\bar{B}^{*}(v, \varepsilon)\left|\frac{g^{2}}{2} \sum_{q}\left(\bar{q} \gamma_{\mu} \gamma_{5} t^{a} q\right)\left(\bar{h} \gamma^{\mu} \gamma_{5} t^{a} h\right)\right| \bar{B}^{*}(v, \varepsilon)\right\rangle=\frac{2 \pi \alpha_{s}}{9} f_{B}^{2} m_{B} \\
& \frac{1}{2 m_{B}}\left\langle\bar{B}^{*}(v, \varepsilon)\left|\frac{g^{2}}{2} \sum_{q}\left(\bar{q} \gamma_{\mu} \gamma_{5} q\right)\left(\bar{h} \gamma^{\mu} \gamma_{5} h\right)\right| \bar{B}^{*}(v, \varepsilon)\right\rangle=\frac{\pi \alpha_{s}}{6} f_{B}^{2} m_{B} .
\end{aligned}
$$

We used here the following relations which are valid in the heavy mass limit

$$
\begin{aligned}
& \left\langle\bar{B}(v)\left|\bar{h} \gamma_{\mu} \gamma_{5} q\right| 0\right\rangle=i f_{B} m_{B} v_{\mu} \\
& \left\langle\bar{B}^{*}(v, \varepsilon)\left|\bar{h} \gamma_{\mu} q\right| 0\right\rangle=f_{B} m_{B} \varepsilon_{\mu}^{*} .
\end{aligned}
$$

Combining these relations one obtains the following expression for the hyperfine splitting

$$
\begin{aligned}
m_{B^{*}}-m_{B}= & -\frac{1}{2 m_{b}} c_{m}(\mu) \frac{4 \mu_{G}^{2}}{3}+\frac{4}{3\left(2 m_{b}\right)^{2}}\left(c_{S O}(\mu) \mu_{S O}^{3}+c_{k}(\mu) c_{m}(\mu) \mu_{\pi G}^{3}\right. \\
& \left.+\left(c_{m}(\mu)\right)^{2} \rho_{A}^{3}-c_{2}^{h l}(\mu) \frac{2 \pi \alpha_{s}}{3} f_{B}^{2} m_{B}-c_{4}^{h l}(\mu) \frac{\pi \alpha_{s}}{2} f_{B}^{2} m_{B}\right)+\mathcal{O}\left(1 / m_{b}^{3}\right) .
\end{aligned}
$$




\begin{tabular}{|c|c|c|c|c|}
\hline & \multicolumn{2}{|c|}{ LLA } & \multicolumn{2}{c|}{ One-loop } \\
\cline { 2 - 5 } & $\mu=0.5 \mathrm{GeV}$ & $\mu=1 \mathrm{GeV}$ & $\mu=0.5 \mathrm{GeV}$ & $\mu=1 \mathrm{GeV}$ \\
\hline \hline$c_{2}^{h l}\left(\mu / m_{c}\right)$ & -0.268 & -0.074 & -0.653 & -0.503 \\
\hline$c_{4}^{h l}\left(\mu / m_{c}\right)$ & -0.117 & -0.038 & -0.348 & -0.268 \\
\hline$c_{2}^{h l}\left(\mu / m_{b}\right)$ & -0.401 & -0.232 & -0.577 & -0.483 \\
\hline$c_{4}^{h l}\left(\mu / m_{b}\right)$ & -0.156 & -0.105 & -0.308 & -0.268 \\
\hline
\end{tabular}

Table 1. The coefficients of the spin-symmetry violating four-quark operators for the charm and bottom cases, at two values of the factorization scale $\mu=0.5$ $\mathrm{GeV}$ and $\mu=1 \mathrm{GeV}$.

This relation can be written only in terms of observable quantities by expressing the quark mass in terms of the pseudoscalar meson mass and multiplying with $m_{B}+m_{B^{*}}$

$$
\begin{aligned}
m_{B^{*}}^{2}-m_{B}^{2}= & -c_{m}(\mu) \frac{4 \mu_{G}^{2}}{3}+\frac{4}{3\left(2 m_{B}\right)}\left(-2 \bar{\Lambda} c_{m}(\mu) \mu_{G}^{2}+c_{S O}(\mu) \mu_{S O}^{3}+c_{k}(\mu) c_{m}(\mu) \mu_{\pi G}^{3}\right. \\
& \left.+\left(c_{m}(\mu)\right)^{2} \rho_{A}^{3}-c_{2}^{h l}(\mu) \frac{2 \pi \alpha_{s}}{3} f_{B}^{2} m_{B}-c_{4}^{h l}(\mu) \frac{\pi \alpha_{s}}{2} f_{B}^{2} m_{B}\right)+\mathcal{O}\left(1 / m_{B}^{2}\right)
\end{aligned}
$$

In Table 1 we present values for the coefficients of the two four-quark operators which contribute to the hyperfine splittings. They are given in both the leading-log approximation and the "full" one-loop form at two different values of the factorization scale $\mu=0.5$ and $1 \mathrm{GeV}$. In computing these values we used $\Lambda_{Q C D}=250 \mathrm{MeV}$ and $m_{c}=1.39 \mathrm{GeV}$, $m_{b}=4.8 \mathrm{GeV}$. For the bottom hadron case we neglected the change in the running of $\alpha_{s}$ across the charm threshold, which gives a negligible error. The one-loop result suggests that the leading-log approximation is very poor for the charm case, where the logarithmic term accounts for at most 30\% of the total correction. The situation is slightly better in the bottom quarks, where its contribution is enhanced to about $50 \%$.

For the purpose of illustration we show in Table 2 the combined contributions of these two operators to the hyperfine splitting of the $\mathrm{D}$ and $\mathrm{B}$ mesons using for their coefficients both approximation methods. For the reasons discussed above we tend to favor the full 
one-loop results over those obtained from the leading log approximation. In computing the matrix elements of the operators in Table 2 we have used the hadronic parameters $f_{D}=170$ $\mathrm{MeV}, f_{B}=180 \mathrm{MeV}, m_{D}=1.97 \mathrm{GeV}, m_{B}=5.28 \mathrm{GeV}$.

\begin{tabular}{|c|c|c|c|c|c|c|}
\hline$\mu_{\text {fact }}$ & $\left\langle\mathcal{O}_{2}^{h l}\right\rangle_{D^{*}-D}$ & $\left\langle\mathcal{O}_{4}^{h l}\right\rangle_{D^{*}-D}$ & $\left\langle\mathcal{O}_{2}^{h l}\right\rangle_{B^{*}-B}$ & $\left\langle\mathcal{O}_{4}^{h l}\right\rangle_{B^{*}-B}$ & $\Delta_{D}(\mathrm{MeV})$ & $\Delta_{B}(\mathrm{MeV})$ \\
\hline \hline $0.5 \mathrm{GeV}$ & $(543 \mathrm{MeV})^{3}$ & $(493 \mathrm{MeV})^{3}$ & $(783 \mathrm{MeV})^{3}$ & $(712 \mathrm{MeV})^{3}$ & $\begin{array}{c}7.4(\mathrm{LLA}) \\
18.9(1-\mathrm{l} .)\end{array}$ & $\begin{array}{c}2.7(\mathrm{LLA}) \\
4.2(1-\mathrm{l} .)\end{array}$ \\
\hline $1.0 \mathrm{GeV}$ & $(431 \mathrm{MeV})^{3}$ & $(392 \mathrm{MeV})^{3}$ & $(622 \mathrm{MeV})^{3}$ & $(565 \mathrm{MeV})^{3}$ & $\begin{array}{c}1.1(\mathrm{LLA}) \\
7.3(1-\mathrm{l} .)\end{array}$ & $\begin{array}{c}0.8(\mathrm{LLA}) \\
1.8(1-1 .)\end{array}$ \\
\hline
\end{tabular}

Table 2. Matrix elements of the four-quark operators $\mathcal{O}_{2}^{h l}$ and $\mathcal{O}_{4}^{h l}$ in the factorization approximation for two different values of the factorization scale $\mu=0.5$ and $1 \mathrm{GeV}$. In the last two columns the total contribution of these two operators to the hyperfine splittings of the $D$ and $B$ mesons $\Delta_{i}=m_{H_{i}^{*}}-m_{H_{i}}$ is shown in both the leading-log approximation and using the one-loop result (1-1.) for their coefficients.

The relation (71) contains the as yet unknown parameters $\mu_{S O}^{3}, \mu_{\pi G}^{3}$ and $\rho_{A}^{3}$. The first parameter vanishes exactly for $S$-wave mesons in potential models as the corresponding operator (6) corresponds to the spin-orbit interaction energy of the heavy quark [6]. The remaining two parameters are not easy to estimate in a model-independent way. The contributions of the four-quark operators are shown in the last two columns of Table 2. Their total contribution is positive and amounts to about $10-20 \mathrm{MeV}$ in the charm case and 1-5 $\mathrm{MeV}$ in the bottom case.

Comparing with the measured hyperfine splittings one can see that a positive $1 / m_{B}$ correction to (71) agrees with the sign of the correction to the heavy mass scaling law. Unfortunately, the lack of information on the precise values of $\mu_{S O}^{3}, \mu_{\pi G}^{3}, \rho_{A}^{3}$ prevents us from making a more quantitative analysis of the deviations from leading order scaling.

Finally we note the relevance of these corrections for the model-independent determination of the matrix element $\mu_{G}^{2}$ from the measured hyperfine splittings in the B system. From (71) we obtain $0.358 \mathrm{GeV}^{2}=\mu_{G}^{2}+0.026+0.021+$ terms proportional to $\mu_{S O}^{3} / m_{B}$ 
and $\mu_{\pi G}^{3} / m_{B}$. The two numbers on the right-hand side correspond to $\bar{\Lambda} \mu_{G}^{2}$ and respectively to the four-quark operators' contribution. In this estimate we used $\bar{\Lambda}=400 \mathrm{MeV}$ and $\mu_{G}^{2}$ $=0.35 \mathrm{GeV}^{2}$. This shows that the theoretical error of this determination coming from the $1 / m_{B}$ terms in (171) may be as high as $13 \%$.

\section{Acknowledgments}

D. P. is grateful to A. Czarnecki, C. D. Lü and D. X. Zhang for discussions and acknowledges support of the Deutsche Forschungsgemeinschaft (DFG) while part of this work was done. He thanks A. Manohar for discussions which helped identify errors in a previous version of this paper. J. G. K. acknowledges partial support from BMFT, FRG under contract 06MZ566. J. C. R. acknowledges the support of the Alexander von Humboldt Foundation. 


\section{APPENDIX A}

A complete study of the renormalized HQET Lagrangian at order $1 / m^{2}$ requires also the computation of the coefficients of operators which vanish by the equation of motion of the heavy quark field. There are altogether six such operators: three local operators which will be chosen as in 13

$$
\begin{aligned}
\mathcal{O}_{8} & =\frac{i g}{2} \bar{h} \sigma_{\mu \nu} F^{\mu \nu}(v \cdot D) h+\frac{i g}{2} \bar{h}(v \cdot D) \sigma_{\mu \nu} F^{\mu \nu} h \\
\mathcal{O}_{9} & =i \bar{h} D^{2}(v \cdot D) h+i \bar{h}(v \cdot D) D^{2} h \\
\mathcal{O}_{10} & =\bar{h}(i v \cdot D)^{3} h
\end{aligned}
$$

and three nonlocal operators consisting of time-ordered products of dimension 5 operators

$$
\begin{aligned}
\mathcal{O}_{e e} & =\frac{i}{2} \int \mathrm{d}^{4} x \mathrm{~T}\left[\bar{h}(i v \cdot D)^{2} h\right](x)\left[\bar{h}(i v \cdot D)^{2} h\right](0) \\
\mathcal{O}_{k e} & =i \int \mathrm{d}^{4} x \mathrm{~T}\left[\bar{h}(i D)^{2} h\right](x)\left[\bar{h}(i v \cdot D)^{2} h\right](0) \\
\mathcal{O}_{m e} & =i \int \mathrm{d}^{4} x \mathrm{~T}\left[\bar{h} \frac{g}{2} \sigma \cdot F h\right](x)\left[\bar{h}(i v \cdot D)^{2} h\right](0)
\end{aligned}
$$

They will be combined together in a vector $\mathcal{H}_{e}$ defined as

$$
\mathcal{H}_{e}=\operatorname{column}\left(\mathcal{O}_{8}, \mathcal{O}_{9}, \mathcal{O}_{10}, \mathcal{O}_{e e}, \mathcal{O}_{k e}, \mathcal{O}_{m e}\right)
$$

These operators mix only with the operators in $\mathcal{H}(22)$. The renormalized operators $\mathcal{H}$ and $\mathcal{H}_{e}$ satisfy a renormalization group equation which can be written in matrix form as

$$
\mu \frac{\mathrm{d}}{\mathrm{d} \mu}\left(\begin{array}{c}
\mathcal{H} \\
\mathcal{H}_{e}
\end{array}\right)+\left(\begin{array}{cc}
A & G \\
0 & H
\end{array}\right)\left(\begin{array}{c}
\mathcal{H} \\
\mathcal{H}_{e}
\end{array}\right)=0 .
$$


The block $A$ has been given in (25), and the remaining ones are given by

$$
\begin{aligned}
& G=\frac{g^{2}}{(4 \pi)^{2}}\left(\begin{array}{cccccc}
0 & 0 & -\frac{16}{3} & 0 & 0 & 0 \\
3 & 0 & 0 & 0 & 0 & 0 \\
0 & \frac{32}{3} & \frac{128}{3} & 0 & -\frac{32}{3} & 0 \\
-\frac{14}{3} & 0 & 0 & 0 & 0 & -\frac{32}{3} \\
9 & 0 & -\frac{32}{3} & 0 & 0 & 0
\end{array}\right), \\
& H=\frac{g^{2}}{(4 \pi)^{2}}\left(\begin{array}{cccccc}
12 & 0 & 0 & 0 & 0 & 0 \\
0 & 0 & -\frac{32}{3} & 0 & 0 & 0 \\
0 & 0 & \frac{16}{3} & 0 & 0 & 0 \\
0 & 0 & \frac{16}{3} & \frac{32}{3} & 0 & 0 \\
0 & -\frac{16}{3} & -32 & -\frac{64}{3} & \frac{16}{3} & 0 \\
-\frac{2}{3} & 0 & 0 & 0 & 0 & \frac{34}{3}
\end{array}\right) \text {. }
\end{aligned}
$$

At the scale $\mu=m$ the coefficients of the operators in (78) are given by

$$
c_{e}=\operatorname{column}(0,0,0,1,-1,1) \text {. }
$$

Integrating the RG equation (79) with this initial condition one obtains the following expressions for the coefficients of the three local operators in (78) in the leading log approximation

$$
\begin{aligned}
c_{8}(\mu) & =-2 z^{-3 / \beta_{0}}+3 z^{-17 /\left(3 \beta_{0}\right)}-\frac{5}{4} z^{-6 / \beta_{0}}+\frac{1}{4}-\frac{9}{2 \beta_{0}} z^{-6 / \beta_{0}} \ln z \\
c_{9}(\mu) & =3 z^{-8 /\left(3 \beta_{0}\right)}-3 \\
c_{10}(\mu) & =-\frac{3}{5} z^{-6 / \beta_{0}}-\frac{65}{9} z^{-2 / \beta_{0}}+9 z^{-16 /\left(3 \beta_{0}\right)}+\frac{23}{30} z^{-8 /\left(3 \beta_{0}\right)}-\frac{35}{18} .
\end{aligned}
$$

We denoted here $z=\left(\alpha_{s}(\mu) / \alpha_{s}(m)\right)$. In deriving (85) we used the result derived in Sec. III for the Wilson coefficient of the Darwin term. It is important to emphasize that these coefficients are (just as $c_{e}(\mu)$ in (5 $)$ ) gauge dependent. Their expressions given above correspond to the Feynman gauge. 


\section{APPENDIX B}

The matching relation connecting the heavy quark field $Q$ in QCD with the effective theory field $h$ is $[12,13]$

$$
Q(x)=\Omega \exp (-i m v \cdot x \psi) h(x)
$$

with

$$
\Omega=\exp \left(\frac{i \not D_{\perp}}{2 m}\right) \exp \left(\frac{1}{4 m^{2}}\left[\left(i \not D_{\|}\right)\left(i \not D_{\perp}\right)+\left(i \not D_{\perp}\right)\left(i \not D_{\|}\right)\right]\right) \cdots
$$

and $\not_{\|}=v \cdot D \psi, D_{\perp}=\not D-\not D_{\|}$. The field $h$ contains both "upper" and "lower" components $h_{ \pm}$, satisfying $\psi h_{ \pm}= \pm h_{ \pm}$. One has

$$
\exp (-i m v \cdot x \psi) h=e^{-i m v \cdot x} \frac{1+\psi}{2} h+e^{i m v \cdot x} \frac{1-\psi}{2} h=e^{-i m v \cdot x} h_{+}+e^{i m v \cdot x} h_{-} .
$$

It can be shown [12] that the field transformation (87) decouples the two components $h_{+}$ and $h_{-}$. The HQET Lagrangian (1/3) used in the main text refers only to the $h_{+}$part.

The most general form for the $1 / \mathrm{m}^{2}$ term in the HQET Lagrangian includes, in addition to the operators introduced so far, also the operators

$$
\begin{aligned}
\mathcal{O}_{8} & =\frac{i g}{2} \bar{h} \sigma_{\mu \nu} F^{\mu \nu}(v \cdot D) h+\frac{i g}{2} \bar{h}(v \cdot D) \sigma_{\mu \nu} F^{\mu \nu} h \\
\mathcal{O}_{9} & =i \bar{h} D^{2}(v \cdot D) h+i \bar{h}(v \cdot D) D^{2} h \\
\mathcal{O}_{10} & =\bar{h}(i v \cdot D)^{3} h .
\end{aligned}
$$

Their renormalization properties are studied in the Appendix A.

The purpose of this Appendix is to show that requiring the invariance of the HQET Lagrangian (II) under a small change in the velocity $v$ (the so-called reparametrization invariance [16]) fixes the coefficients $c_{S O}(\mu)$ [14] and $c_{9}(\mu)$. However, no constraint for $c_{D}(\mu)$ is obtained in this way, in contrast to [14].

We start by computing the change in the $h_{+}$field under an infinitesimal change of the velocity parameter $v \rightarrow v^{\prime}=v+\Delta v$. This can be obtained from (86) by taking into account the invariance of the QCD field under this transformation $\delta Q=0$ as

$$
\delta h_{+}=\delta\left[\frac{1+\psi}{2} \exp (i m v \cdot x) \Omega^{-1}\right] \Omega \exp (-i m v \cdot x \psi) h .
$$


Explicitly, to first order in $\Delta v$ and up to second order in $1 / m$,

$$
\delta h_{+}=\Delta v^{\mu}\left\{i m x_{\mu}+\frac{1}{2} \gamma_{\mu}+\frac{i}{4 m}\left[\gamma_{\mu} D_{\perp}+D_{\mu}\right]+\mathcal{O}\left(1 / m^{2}\right)\right\} h_{+} .
$$

Note that no negative component field $h_{-}$is introduced by this transformation.

The variation of the HQET Lagrangian (10 (13) is obtained by the variation of the effective field (93) and the variation of the $v$-dependent operators, including the operators (89.91). After some algebra one obtains

$$
\begin{aligned}
\delta \mathcal{L} & =\left(1-c_{k}(\mu)\right) \bar{h}_{+} i \Delta v \cdot D h_{+}-\frac{1}{m}\left\{-\left(c_{e}+c_{9}-1\right) \bar{h}_{+}(\Delta v \cdot D)(v \cdot D) h_{+}\right. \\
& -\frac{1}{4}\left(c_{e}+1+c_{9}+c_{m}+\frac{c_{S O}+1}{2}\right) \bar{h}_{+} i g \gamma \cdot \Delta v \gamma^{\nu} v^{\mu} F_{\mu \nu} h_{+} \\
& \left.-\frac{1}{4}\left(c_{e}+1+c_{9}-c_{m}-\frac{c_{S O}+1}{2}\right) \bar{h}_{+} i g \gamma^{\nu} \gamma \cdot \Delta v v^{\mu} F_{\mu \nu} h_{+} \mathcal{O}\left(1 / m^{2}\right)\right\} .
\end{aligned}
$$

This will vanish for any $\Delta v$ provided that the following identities hold

$$
\begin{aligned}
c_{k}(\mu) & =1 \\
c_{9}(\mu) & =-1-c_{e}(\mu) \\
c_{S O}(\mu) & =-1-2 c_{m}(\mu) .
\end{aligned}
$$

The first constraint has been given in [16] and is perhaps the best known application of the reparametrization invariance principle. The constraint (97) has been presented in [14] and its prediction for $c_{S O}$ agrees with the explicit calculation in [13] (see (46)). The relation (96)) together with (44) predicts the following value for $c_{9}(\mu)$

$$
c_{9}(\mu)=3\left[\left(\frac{\alpha_{s}(\mu)}{\alpha_{s}(m)}\right)^{-8 /\left(3 \beta_{0}\right)}-1\right] .
$$

This agrees with the expression of this coefficient obtained by direct computation in leading $\log$ approximation (84). 
[1] N. Isgur and M.B. Wise, in Stone, S. (ed.), B physics, World Scientific, 1994.

[2] M. Neubert, Phys. Rep. 245 (1994) 259.

[3] M. Shifman, TPI-MINN-95-31-T, 1995.

[4] A. Falk and M. Neubert, Phys. Rev. D 47 (1993) 2965.

[5] T. Mannel, Phys. Rev. D 50 (1994) 428.

[6] I. Bigi, M. Shifman, N.G. Uraltsev and A. Vainshtein, Phys. Rev. D 52 (1995) 196.

[7] B. Blok, R. D. Dikeman and M. A. Shifman, Phys. Rev. D 51, 6167 (1995).

[8] M. Gremm and A. Kapustin, Phys. Rev. D 55, 6924 (1997).

[9] M. E. Luke, Phys. Lett. B 252 (1990) 447.

[10] E. Eichten and B. Hill, Phys. Lett. B 234 (1990) 511.

[11] A. Falk, B. Grinstein and M. Luke, Nucl. Phys. B 357 (1991) 185.

[12] J. G. Körner and G. Thompson, Phys. Lett. B 264 (1991) 185.

[13] S. Balk, J.G. Körner and D. Pirjol, Nucl. Phys.B 428 (1994) 499.

[14] Y.Q. Chen, Y.P. Kuang and R. Oakes, Phys. Rev. D52 (1995) 264.

[15] C. Balzereit and T. Ohl, Phys. Lett. B 386, 335 (1996) arXiv:hep-ph/9604352.

[16] M. Luke and A. Manohar, Phys. Lett. B286 (1992) 348.

[17] V. Novikov, M. Shifman, A. Vainshtein and V. Zakharov, Fortschr. Phys. 32 (1984) 585.

[18] P. L. Cho and E. H. Simmons, Phys. Lett. B 323, 401 (1994).

[19] P. L. Cho and E. H. Simmons, Phys. Rev. D 51, 2360 (1995).

[20] M. Shifman, A. Vainshtein and V. Zakharov, Nucl. Phys. B147 (1986) 385.

[21] M. Jamin and M. Kremer, Nucl. Phys. B 277, (1986) 349.

[22] D. Barfoot and D. Broadhurst, Phys. Lett. B 166 (1986) 347.

[23] A. V. Manohar, Phys. Rev. D 56, 230 (1997).

[24] A.J. Buras, E. Floratos, D. Ross and C. Sachrajda, Nucl. Phys. B 131 (1977) 308.

[25] D. Broadhurst and A.G. Grozin, Phys. Rev. D 52 (1995) 4082.

[26] I. Bigi, M. Shifman, N. Uraltsev and A. Vainshtein, Int. J. Mod. Phys. A 9 (1994) 2467.

[27] C.K. Chow, Phys. Rev. D 51 (1995) 3587.

[28] C.K. Chow and D. Pirjol, Phys. Rev.D 53 (1996) 3998.

[29] N. Di Bartolomeo, R. Gatto, F. Feruglio and G. Nardulli, Phys. Lett.B 347 (1995) 405. 
[30] C. W. Bauer and A. V. Manohar, Phys. Rev. D 57, 337 (1998).

[31] It also agrees with the sum $c_{D}+c_{1}^{h l}$ of the Eqs. (27),(28) in the v1 of the present paper. 